\title{
Weighted Composition Operators Between Spaces of Dirichlet Type
}

\author{
Sanjay KUMAR \\ Department of Mathematics \\ University of Jammu \\ Jammu-180 006 — India. \\ sanjaykmath@gmail.com
}

Received: January 27, 2008

Accepted: October 14, 2008

\begin{abstract}
In this work we characterize boundedness and compactness of weighted composition operators acting between Dirichlet type spaces by using Carleson measures. We also find essential norm estimates for these operators.

Key words: Bergman spaces, Carleson measure, essential norm, spaces of Dirichlet type, weighted compositon operators.

2000 Mathematics Subject Classification: Primary 47B33, 46E30; Secondary 47B07, $46 \mathrm{~B} 70$.
\end{abstract}

\section{Introduction}

Let $\mathbf{D}$ denote the open unit disk in the complex plane $\mathbf{C}$. We will use the notation $H(\mathbf{D})$ for the space of complex valued holomorphic functions on the open unit disk $\mathbf{D}$. Let $\varphi, \psi \in H(\mathbf{D})$ be such that $\varphi(\mathbf{D}) \subseteq \mathbf{D}$. Then the weighted composition operator $W_{\varphi, \psi}$ acting on $H(\mathbf{D})$ is defined as

$$
W_{\varphi, \psi}(f)(z)=\psi(z) f(\varphi(z))
$$

When $\psi \equiv 1$, we just have the composition operator $C_{\varphi}$, defined by

$$
C_{\varphi}(f)=f \circ \varphi
$$

Also if $\varphi \equiv I$, the identity function, then we get the multiplication operator $M_{\psi}$ defined by $M_{\psi}(f)(z)=\psi(z) f(z)$. 
Weighted composition operators are a general class of operators and they appear naturally in the study of surjective isometries on most of the function spaces, semigroup theory, dynamical systems, Brennan's conjecture, etc.

In this paper, we study the weighted composition operators between spaces of Dirichlet type $\mathcal{D}_{\alpha}^{p}$. We also obtain estimates for the essential norm of weighted composition operators on these spaces. For weighted composition operators on spaces of holomorphic functions one can refer to $[2,4,11,17,20,23]$ and the references therein.

For any $a \in \mathbf{D}$, define

$$
\sigma_{a}(z)=\frac{a-z}{1-\bar{a} z}, z \in \mathbf{D},
$$

where $\sigma_{a}(z)$ is the Möbius transformation of $\mathbf{D}$. Also

$$
\left|\sigma_{a}^{\prime}(z)\right|=\frac{1-|a|^{2}}{|1-\bar{a} z|^{2}}
$$

and

$$
1-\left|\sigma_{a}(z)\right|^{2}=\frac{\left(1-|a|^{2}\right)\left(1-|z|^{2}\right)}{|1-\bar{a} z|^{2}}=\left(1-|z|^{2}\right)\left|\sigma_{a}^{\prime}(z)\right|,
$$

for all $a, z \in \mathbf{D}$.

Let $d A(z)=\frac{1}{\pi} d x d y$ denote the normalised Lebesgue area measure on $\mathbf{D}$. Also, let $d A_{\alpha}(z)=(1+\alpha)^{\pi}\left(1-|z|^{2}\right)^{\alpha} d A(z)$ denote the weighted Lebesgue area measure on $\mathbf{D}$, where $-1<\alpha<\infty$. For $0<p<\infty$ and $-1<\alpha<\infty$, the weighted Bergman space $L_{a}^{p, \alpha}$ consists of those functions $f$ holomorphic on $\mathbf{D}$ such that

$$
\|f\|_{L_{a}^{p, \alpha}}=\left(\int_{\mathbf{D}}|f(z)|^{p} d A_{\alpha}(z)\right)^{1 / p}<\infty .
$$

If $\alpha=0$, we get the Bergman space $L_{a}^{p}$.

For $0<p<\infty$ and $-1<\alpha<\infty$, the spaces of Dirichlet type $\mathcal{D}_{\alpha}^{p}$ consist of those functions $f$ holomorphic on $\mathbf{D}$ such that

$$
\|f\|_{\mathcal{D}_{\alpha}^{p}}=\left(|f(0)|^{p}+\int_{\mathbf{D}}\left|f^{\prime}(z)\right|^{p} d A_{\alpha}(z)\right)^{1 / p}<\infty .
$$

That is, $f \in \mathcal{D}_{\alpha}^{p}$ if and only if $f^{\prime} \in L_{a}^{p, \alpha}$. The spaces $\mathcal{D}_{\alpha}^{p}$ are called Dirichlet spaces if $p \geq \alpha+1$. For $\alpha=0$, the space $\mathcal{D}_{0}^{2}$ is the classical Dirichlet space. If $p<\alpha+1$, then it is well known that $\mathcal{D}_{\alpha}^{p}=L_{a}^{p, \alpha-p}$ (see e.g., Theorem 6 of [6]). Also $\mathcal{D}_{1}^{2}$ equals to the Hardy spaces $H^{2}$. Further, $\mathcal{D}_{\alpha}^{p} \subset \mathcal{D}_{\alpha}^{q}$, if $1 \leq q<p$.

\section{Boundedness and compactness}

In this section, we characterize boundedness and compactness of $W_{\varphi, \psi}$ acting on weighted Dirichlet spaces by using Carleson measures. 
Let $\mu$ be a positive Borel measure on $\mathbf{D}$. Let $\mathrm{X}$ be a Banach space of holomorphic functions on $\mathbf{D}$. Let $q>0$. We say that $\mu$ is an $(X, q)$ - Carleson measure if there is a constant $C>0$ such that for any $f \in X$,

$$
\int_{\mathbf{D}}|f(z)|^{q} d \mu(z) \leq C\|f\|_{X}^{q} .
$$

For $s>0$, we say that $\mu$ is an $s$-Carleson measure on $\mathbf{D}$ if there is a positive constant $C$ such that

$$
\mu(S(b, h)) \leq C h^{s},
$$

for all $b \in \partial \mathbf{D}$ and $0<h \leq 2$, where

$$
S(b, h)=\{z \in \overline{\mathbf{D}}:|z-b|<h\} .
$$

A 1-Carleson measure will be simply called a Carleson measure. Similarly, $\mu$ is called a compact s-Carleson measure on $\mathbf{D}$ if

$$
\lim _{h \rightarrow 0} \sup _{b \in \partial \mathbf{D}} \frac{\mu(S(b, h))}{h^{s}}=0 .
$$

Also one can see that (1) and (2) are equivalent to

$$
\sup _{I \subset \partial \mathbf{D}} \frac{\mu(S(I))}{|I|^{s}}<\infty
$$

and

$$
\lim _{|I| \rightarrow 0} \frac{\mu(S(I))}{|I|^{s}}=0,
$$

respectively, where $|I|$ denotes the arc length of I and $S(I)$ denotes the Carleson square based on I, that is

$$
S(I)=\left\{z \in \mathbf{D}: 1-|I| \leq|z|<1, \frac{z}{|z|} \in I\right\} .
$$

A non negative measure $\mu$ on $\mathbf{D}$ is called a Carleson measure for $\mathcal{D}_{\alpha}^{p}$ if there is a constant $C>0$ such that

$$
\int_{\mathbf{D}}|f(z)|^{p} d \mu(z) \leq C\|f\|_{\mathcal{D}_{\alpha}^{p}}^{p},
$$

for all $f \in \mathcal{D}_{\alpha}^{p}$. That is, the inclusion operator $i$ from $\mathcal{D}_{\alpha}^{p}$ into $L^{p}(\mathbf{D}, d \mu)$ is bounded. Again, we call Carleson measure for $\mathcal{D}_{\alpha}^{p}$, a vanishing Carleson measure for $\mathcal{D}_{\alpha}^{p}$ if the inclusion operator $i$ from $\mathcal{D}_{\alpha}^{p}$ into $L^{p}(\mathbf{D}, d \mu)$ is compact.

The following theorem characterizes Carleson measures for $\mathcal{D}_{\alpha}^{p}$. 
Theorem 2.1 ([24, Theorem 1, part(d)]). Suppose that $\alpha>-1$ and $p \geq \alpha+1$. A non negative measure $\mu$ on $\mathbf{D}$ is a Carleson measure for $\mathcal{D}_{\alpha}^{p}$ if and only if there is a constant $C>0$ such that for $1<p=\alpha+1 \leq 2$,

$$
\mu(S(I)) \leq C|I|,
$$

for any $I \subset \partial \mathbf{D}$.

Let $0<p<q<\infty$ and $-1<\alpha<\infty$. Let $\varphi \in \mathcal{D}_{\alpha}^{p}, \psi \in \mathcal{D}_{\alpha}^{q}$ be such that $\varphi$ is self-map of $\mathbf{D}$ and $\psi \varphi^{\prime} \in L_{a}^{q, \alpha}$. Then we define the measures $\mu_{\varphi, \psi \varphi^{\prime}, q}$ and $\nu_{\varphi, \psi^{\prime}, q}$ on D by

$$
\mu_{\varphi, \psi \varphi^{\prime}, q}(E)=\int_{\varphi^{-1}(E)}\left|\psi(z) \varphi^{\prime}(z)\right|^{q} d A_{\alpha}(z)
$$

and

$$
\nu_{\varphi, \psi^{\prime}, q}(E)=\int_{\varphi^{-1}(E)}\left|\psi^{\prime}(z)\right|^{q} d A_{\alpha}(z),
$$

where $\mathrm{E}$ is a measurable subset of the unit disk $\mathbf{D}$. Again for $\psi \in L_{a}^{q, \alpha}$, we define the measure $\nu_{\varphi, \psi, q}$ on $\mathbf{D}$ by

$$
\nu_{\varphi, \psi, q}(E)=\int_{\varphi^{-1}(E)}|\psi(z)|^{q} d A_{\alpha}(z) .
$$

Using [2; 8 , page 163], the following lemma, whose proof is omitted, follows easily.

Lemma 2.2. Take $-1<\alpha<\infty$. Let $\varphi \in \mathcal{D}_{\alpha}^{q}$ be such that $\varphi(\mathbf{D}) \subseteq \mathbf{D}$ and $\psi \in \mathcal{D}_{\alpha}^{q}$. Then

$$
\int_{\mathbf{D}} g(w) d \mu_{\varphi, \psi \varphi^{\prime}, q}(w)=\int_{\mathbf{D}}\left|\psi(z) \varphi^{\prime}(z)\right|^{q}(g \circ \varphi)(z) d A_{\alpha}(z)
$$

and

$$
\int_{\mathbf{D}} g(w) d \nu_{\varphi, \psi^{\prime}, q}(w)=\int_{\mathbf{D}}\left|\psi^{\prime}(z)\right|^{q}(g \circ \varphi)(z) d A_{\alpha}(z),
$$

where $g$ is an arbitrary measurable positive function on $\mathbf{D}$.

We will state several theorems that we need for our work. Precise references are given.

Theorem 2.3 ([10]). Take $0<p<q<\infty$ and $-1<\alpha<\infty$. Let $\mu$ be a positive Borel measure on $\mathbf{D}$. Then $\mu$ is said to be a $\frac{q}{p}(2+\alpha)$-Carleson measure for $L_{a}^{p, \alpha}$ if and only if $L_{a}^{p, \alpha} \subset L^{q}(\mathbf{D}, d \mu)$. In this case the inclusion operator $i$ from $L_{a}^{p, \alpha}$ into $L^{q}(\mathbf{D}, d \mu)$ is bounded.

Theorem 2.4 ([10]). Take $0<p \leq q<\infty$ and $-1<\alpha<\infty$. Let $\mu$ be a positive Borel measure on $\mathbf{D}$. Then $\mu$ is said to be a vanishing $\frac{q}{p}(2+\alpha)$ - Carleson measure for $L_{a}^{p, \alpha}$ if and only if $L_{a}^{p, \alpha} \subset L^{q}(\mathbf{D}, d \mu)$. In this case the inclusion operator $i$ from $L_{a}^{p, \alpha}$ into $L^{q}(\mathbf{D}, d \mu)$ is compact. 
Theorem 2.5 ([4, Theorem 7$])$. Let $\mu$ be a positive measure on $\mathbf{D}$. Let $0<q<p<\infty$ and $-1<\alpha<\infty$. Then the following statements are equivalent:

(i) $\mu$ is a $\left(L_{a}^{p, \alpha}, q\right)$-Carleson measure.

(ii) $B_{\alpha}(\mu) \in L_{a}^{p / p-q, \alpha}$,

where $B_{\alpha}(\mu)=\int_{\mathbf{D}}\left|\sigma_{z}^{\prime}(\omega)\right|^{2+\alpha} d \mu(\omega)$.

Theorem 2.6 ([5, Theorem 1]). Suppose that $0<q<p<\infty,-1<\alpha<\infty$ and let $\mu$ be a positive measure in $\mathbf{D}$. If $p<\alpha+2$, then $\mathcal{D}_{\alpha}^{p} \subset L^{q}(\mathbf{D}, d \mu)$ if and only if $\mu$ is a $\frac{q}{p}(\alpha-p+2)$-Carleson (vanishing Carleson) measure.

Theorem 2.7. Suppose that $0<p<q<\infty,-1<\alpha<\infty$ and let $\mu$ be a positive Borel measure on $\mathbf{D}$. If $p<\alpha+2$, then the following statements are equivalent:

(i) The inclusion $i: \mathcal{D}_{\alpha}^{p} \rightarrow L^{q}(\mathbf{D}, d \mu)$ is bounded.

(ii) The measure $\mu$ is a $\frac{q}{p}(\alpha-p+2)$-Carleson measure, that is, there is a constant $C_{1}<\infty$ such that

$$
\mu(S(I)) \leq C_{1}|I|^{\frac{q}{p}(\alpha-p+2)} .
$$

(iii) There exists a constant $C_{2}<\infty$ such that for any $a \in \mathbf{D}$

$$
\int_{\mathbf{D}}\left|\sigma_{a}^{\prime}(z)\right|^{\frac{q}{p}(\alpha-p+2)} d \mu(z) \leq C_{2}
$$

The above result was proved by several authors. The equivalence of (i) and (ii) can be found in Theorem 1 of [6], and a proof of the equivalence of (ii) and (iii) can be found in [1].

Theorem 2.8. Suppose that $0<p<q<\infty,-1<\alpha<\infty$ and let $\mu$ be a positive Borel measure on D. If $p<\alpha+2$, then the following statements are equivalent:

(i) The inclusion $i: \mathcal{D}_{\alpha}^{p} \rightarrow L^{q}(\mathbf{D}, d \mu)$ is compact.

(ii) The measure $\mu$ is a vanishing $\frac{q}{p}(\alpha-p+2)$-Carleson measure, that is, there is a constant $C_{1}<\infty$ such that

$$
\lim _{I \subset \partial \mathbf{D}|I| \rightarrow 0} \frac{\mu(S(I))}{|I|^{\frac{q}{p}(\alpha-p+2)}}=0 .
$$

(iii) For all $a \in \mathbf{D}$, we have

$$
\lim _{|a| \rightarrow 1} \int_{\mathbf{D}}\left|\sigma_{a}^{\prime}(z)\right|^{\frac{q}{p}(\alpha-p+2)} d \mu(z)=0 .
$$


Lemma 2.9. Suppose that $0<p<q<\infty$ and $-1<\alpha<\infty$. Let $\varphi$ be a holomorphic self-map of $\mathbf{D}$ and let $\psi \in \mathcal{D}_{\alpha}^{q}$ be such that $W_{\varphi, \psi}$ from $\mathcal{D}_{\alpha}^{p}$ into $\mathcal{D}_{\alpha}^{q}$ is bounded.Then $W_{\varphi, \psi}$ from $\mathcal{D}_{\alpha}^{p}$ into $\mathcal{D}_{\alpha}^{q}$ is compact if and only if whenever $\left\{f_{n}\right\}$ is a bounded sequence in $\mathcal{D}_{\alpha}^{p}$ converging to zero uniformly on compact subsets of $\mathbf{D}$, then $\left\|W_{\varphi, \psi}\left(f_{n}\right)\right\|_{\mathcal{D}_{\alpha}^{q}} \rightarrow 0$.

Proof. We know, by hypothesis, that $W_{\varphi, \psi}$ from $\mathcal{D}_{\alpha}^{p}$ into $\mathcal{D}_{\alpha}^{q}$ is bounded. If $\left\{f_{n}\right\}$ is a bounded sequence in $\mathcal{D}_{\alpha}^{p}$, then from the growth estimate, it follows that

$$
\left|f_{n}(z)-f_{n}(0)\right|^{p} \leq C|| f_{n} \|_{\mathcal{D}_{\alpha}^{p}} \log \frac{1}{1-|z|},
$$

for all $z \in \mathbf{D}$. Using [22, Lemma 1.10] and (3), we only need to prove that the closed unit ball of $\mathcal{D}_{\alpha}^{p}$ is a compact subset of $\mathcal{D}_{\alpha}^{p}$ in the topology of uniform convergence on compact subsets of $\mathbf{D}$. Let $\left\{f_{n}\right\}$ be a sequence in the closed unit ball of $\mathcal{D}_{\alpha}^{p}$, then from $(3),\left\{f_{n}\right\}$ is uniformly bounded on compact subset of D. By Montel's Theorem [3, page 137], there is a subsequence $\left\{f_{n_{k}}\right\}$ and an analytic function $g$ such that $f_{n_{k}} \rightarrow g$ uniformly on compact subsets of $\mathbf{D}$. We show that $g \in \mathcal{D}_{\alpha}^{q}$ :

$$
\begin{aligned}
\int_{\mathbf{D}}\left|g^{\prime}(z)\right|^{q}\left(1-|z|^{2}\right)^{\alpha} d A(z) & =\int_{\mathbf{D}} \lim _{k \rightarrow \infty}\left|f_{n_{k}}^{\prime}(z)\right|^{q}\left(1-|z|^{2}\right)^{\alpha} d A(z) \\
& \leq \lim _{k \rightarrow \infty} \inf \int_{\mathbf{D}}\left|f_{n_{k}}^{\prime}(z)\right|^{q}\left(1-|z|^{2}\right)^{\alpha} d A(z) \\
& \leq \lim _{k \rightarrow \infty} \inf \left\|f_{n_{k}}\right\|_{\mathcal{D}_{\alpha}^{p}}^{q},
\end{aligned}
$$

by Fatou's lemma. This gives $g \in \mathcal{D}_{\alpha}^{q}$.

Now, we can prove the following theorem.

Theorem 2.10. Suppose that $0<p<q<\infty$ and $-1<\alpha<\infty$. Let $\varphi \in \mathcal{D}_{\alpha}^{p}$ be such that $\varphi$ is a self-map of $\mathbf{D}$ and $\psi \in L_{a}^{q, \alpha}$. For $p<\alpha+2$, if the measure $\nu_{\varphi, \psi, q}$ is a vanishing $\frac{q}{p}(\alpha-p+2)$-Carleson measure, then the operator $W_{\varphi, \psi}$ from $\mathcal{D}_{\alpha}^{p}$ into $L_{a}^{q, \alpha}$ is bounded. Moreover, $W_{\varphi, \psi}$ from $\mathcal{D}_{\alpha}^{p}$ into $L_{a}^{q, \alpha}$ is compact.

Proof. We need to show only compactness. For this, let $\left\{f_{n}\right\}$ be a bounded sequence in $L_{a}^{p, \alpha}$ such that $f_{n} \rightarrow 0$ uniformly on compact subsets of $\mathbf{D}$ as $n \rightarrow \infty$. Suppose $g_{n} \in \mathcal{D}_{\alpha}^{p}$ such that $g_{n}^{\prime}=f_{n}$ and $g_{n}(0)=0$ for every $n$. Then the sequence $\left\{g_{n}\right\}$ also converges to zero uniformly on compact subsets of $\mathbf{D}$ as $n \rightarrow \infty$. Now, we have

$$
\begin{aligned}
\left\|W_{\varphi, \psi}\left(g_{n}\right)\right\|_{L_{a}^{q, \alpha}}^{q} & =\int_{\mathbf{D}}|\psi(z)|^{q}\left|\left(g_{n} \circ \varphi\right)(z)\right|^{q} d A_{\alpha}(z) \\
& =\int_{\mathbf{D}}\left|g_{n}(w)\right|^{q} d \nu_{\varphi, \psi, q}(w) \rightarrow 0, \quad \text { as } n \rightarrow \infty .
\end{aligned}
$$

Thus $W_{\varphi, \psi}: \mathcal{D}_{\alpha}^{p} \rightarrow L_{a}^{q, \alpha}$ is compact. The proof is finished. 
Remark 2.11. Using results from [7], Theorem 2.10 can be proved for the following cases by adjusting the Carleson measure:

(a) For $0<p<q<\infty$ and $\alpha=p-1$, we have to take $\nu_{\varphi, \psi, q}$ as a vanishing $q / p$-Carleson measure.

(b) If $0<p \leq 2$ and $\alpha=p-1$, then we have to choose $\nu_{\varphi, \psi, q}$ as a vanishing 1-Carleson measure.

(c) For $0<p \leq 1,-1<\alpha<p-1$ and $p<q<\infty$, we can take $\nu_{\varphi, \psi, q}$ as a vanishing $\frac{q}{p}(\alpha-p+2)-$ Carleson measure.

(d) If $\alpha+1<p<\alpha+2$, then we can take $\nu_{\varphi, \psi, q}$ as a vanishing $\frac{q}{p}(\alpha-p+2)-$ Carleson measure.

Theorem 2.12. Take $0<p<q<\infty$ and $-1<\alpha<\infty$. Let $\varphi \in \mathcal{D}_{\alpha}^{p}$ be such that $\varphi$ is a self-map of $\mathbf{D}$ and let $\psi \in \mathcal{D}_{\alpha}^{q}$. For $p<\alpha+2$, suppose that the measure $\nu_{\varphi, \psi^{\prime}, q}$ is a $\frac{q}{p}(\alpha-p+2)$-Carleson measure. Then the operator $W_{\varphi, \psi}$ from $\mathcal{D}_{\alpha}^{p}$ into $\mathcal{D}_{\alpha}^{q}$ is bounded if and only if $W_{\varphi, \psi \varphi^{\prime}}$ from $L_{a}^{p, \alpha}$ into $L_{a}^{q, \alpha}$ is bounded.

Proof. Suppose $W_{\varphi, \psi}$ from $\mathcal{D}_{\alpha}^{p}$ into $\mathcal{D}_{\alpha}^{q}$ is bounded. Then there exists a constant $C_{1}>0$ such that

$$
\left\|W_{\varphi, \psi}(f)\right\|_{\mathcal{D}_{\alpha}^{q}}^{q} \leq C_{1}\|f\|_{\mathcal{D}_{\alpha}^{p}}^{q} \text { for all } f \in \mathcal{D}_{\alpha}^{p}
$$

Also, by Theorem 2.10, we can find a constant $C_{2}>0$ such that

$$
\left\|W_{\varphi, \psi^{\prime}}(f)\right\|_{L_{a}^{q, \alpha}}^{q} \leq C_{2}\|f\|_{\mathcal{D}_{\alpha}^{p}}^{q} \text { for all } f \in \mathcal{D}_{\alpha}^{p} .
$$

Take $f \in L_{a}^{p, \alpha}$. Let $g \in \mathcal{D}_{\alpha}^{p}$ be such that $g^{\prime}=f$ and $g(0)=0$. We have

$$
\begin{aligned}
\left\|W_{\varphi, \psi \varphi^{\prime}}(f)\right\|_{L_{a}^{q, \alpha}}^{q} & =\left\|\psi \varphi^{\prime} f \circ \varphi\right\|_{L_{a}^{q, \alpha}}^{q} \\
& =\left\|\psi \varphi^{\prime} f \circ \varphi+\psi^{\prime} g \circ \varphi-\psi^{\prime} g \circ \varphi\right\|_{L_{a}^{q, \alpha}}^{q} \\
& \leq\left\|(\psi g \circ \varphi)^{\prime}\right\|_{L_{a}^{q, \alpha}}^{q}+\left\|\psi^{\prime} g \circ \varphi\right\|_{L_{a}^{q, \alpha}}^{q} \\
& =\left\|W_{\varphi, \psi}(g)\right\|_{\mathcal{D}_{\alpha}^{q}}^{q}+\left\|W_{\varphi, \psi^{\prime}}(g)\right\|_{L_{a}^{q, \alpha}}^{q} \\
& \leq\left(C_{1}+C_{2}\right)\|g\|_{\mathcal{D}_{\alpha}^{p}}^{q}=\left(C_{1}+C_{2}\right)\|f\|_{L_{a}^{p, \alpha}}^{q} .
\end{aligned}
$$

This implies that $W_{\varphi, \psi \varphi^{\prime}}: L_{a}^{p, \alpha} \rightarrow L_{a}^{q, \alpha}$ is bounded.

Conversely, suppose that the operator $W_{\varphi, \psi \varphi^{\prime}}$ from $L_{a}^{p, \alpha}$ into $L_{a}^{q, \alpha}$ is bounded. Take $f \in \mathcal{D}_{\alpha}^{p}$ such that $f(0)=0$. Then we have

$$
\begin{aligned}
\left\|W_{\varphi, \psi}(f)\right\|_{\mathcal{D}_{\alpha}^{q}}^{q} & =\left\|(\psi f \circ \varphi)^{\prime}\right\|_{L_{a}^{q, \alpha}}^{q} \\
& =\left\|\psi \varphi^{\prime} f^{\prime} \circ \varphi+\psi^{\prime} f \circ \varphi\right\|_{L_{a}^{q, \alpha}}^{q} \\
& \leq\left\|W_{\varphi, \psi \varphi^{\prime}}\left(f^{\prime}\right)\right\|_{L_{a}^{q, \alpha}}^{q}+\left\|W_{\varphi, \psi^{\prime}}(f)\right\|_{L_{a}^{q, \alpha}}^{q}<\infty .
\end{aligned}
$$


Theorem 2.13. Suppose that $0<p<q<\infty$ and $-1<\alpha<\infty$. Let $\varphi \in \mathcal{D}_{\alpha}^{p}$ be such that $\varphi$ is a self-map of $\mathbf{D}$ and let $\psi \in \mathcal{D}_{\alpha}^{q}$. For $p<\alpha+2$, suppose that the measure $\nu_{\varphi, \psi^{\prime}, q}$ is a $\frac{q}{p}(\alpha-p+2)$-Carleson measure. Then the operator $W_{\varphi, \psi}$ from $\mathcal{D}_{\alpha}^{p}$ into $\mathcal{D}_{\alpha}^{q}$ is bounded if and only if

$$
\sup _{a \in \mathbf{D}} \int_{\mathbf{D}}\left(\frac{1-|a|^{2}}{|1-\bar{a} \omega|^{2}}\right)^{\frac{q}{p}(2+\alpha)} d \mu_{\varphi, \psi \varphi^{\prime}, q}(\omega)<\infty .
$$

Proof. Suppose that $W_{\varphi, \psi}$ from $\mathcal{D}_{\alpha}^{p}$ into $\mathcal{D}_{\alpha}^{q}$ is bounded. Then by Theorem 2.12, $W_{\varphi, \psi \varphi^{\prime}}$ from $L_{a}^{p, \alpha}$ into $L_{a}^{q, \alpha}$ is bounded. This means that their exists a constant $C>0$ such that

$$
\left\|W_{\varphi, \psi \varphi^{\prime}}(f)\right\|_{L_{a}^{q, \alpha}} \leq C\|f\|_{L_{a}^{p, \alpha}} .
$$

That is,

$$
\int_{\mathbf{D}}\left|\psi(z) \varphi^{\prime}(z)\right|^{q}|(f \circ \varphi)(z)|^{q} d A_{\alpha}(z) \leq C\|f\|_{L_{a}^{p, \alpha}}^{q} \text { for all } f \in L_{a}^{p, \alpha} .
$$

Therefore by Lemma 2.2, we get

$$
\int_{\mathbf{D}}|f(w)|^{q} d \mu_{\varphi, \psi \varphi^{\prime}, q}(w) \leq C\|f\|_{L_{a}^{p, \alpha}}^{q} .
$$

So by using Theorem A of [5], we get that

$$
\sup _{a \in \mathbf{D}} \int_{\mathbf{D}}\left(\frac{1-|a|^{2}}{|1-\bar{a} \omega|^{2}}\right)^{\frac{q}{p}(2+\alpha)} d \mu_{\varphi, \psi \varphi^{\prime}, q}(\omega)<\infty .
$$

Theorem 2.14. Suppose that $0<p<q<\infty$ and $-1<\alpha<\infty$. Let $\varphi \in \mathcal{D}_{\alpha}^{p}$ be such that $\varphi$ is a self-map of $\mathbf{D}$ and let $\psi \in \mathcal{D}_{\alpha}^{q}$. For $p<\alpha+2$, if the measure $\nu_{\varphi, \psi^{\prime}, q}$ is a $\frac{q}{p}(\alpha-p+2)$-Carleson measure, then the operator $W_{\varphi, \psi}$ from $\mathcal{D}_{\alpha}^{p}$ into $\mathcal{D}_{\alpha}^{q}$ is compact if and only if $W_{\varphi, \psi \varphi^{\prime}}$ from $L_{a}^{p, \alpha}$ into $L_{a}^{q, \alpha}$ is compact.

Proof. First suppose that $W_{\varphi, \psi}$ from $\mathcal{D}_{\alpha}^{p}$ into $\mathcal{D}_{\alpha}^{q}$ is compact. Let $\left\{f_{n}\right\}$ be a bounded sequence in $L_{a}^{p, \alpha}$ such that $f_{n}$ converges to zero uniformly on compact subsets of $\mathbf{D}$ as $n \rightarrow \infty$. Let $g_{n} \in \mathcal{D}_{\alpha}^{p}$ be such that $g_{n}^{\prime}=f_{n}$ and $g_{n}(0)=0$ for every $n$. Then the sequence $\left\{g_{n}\right\}$ also converges to zero uniformly on compact subsets of $\mathbf{D}$ as $n \rightarrow \infty$. Also, $W_{\varphi, \psi}$ from $\mathcal{D}_{\alpha}^{p}$ into $\mathcal{D}_{\alpha}^{q}$ is compact. This implies that $\left\|W_{\varphi, \psi}\left(g_{n}\right)\right\|_{\mathcal{D}_{\alpha}^{q}}^{q}$ converges to 0 as $n \rightarrow \infty$. Again, by Theorem $2.10, W_{\varphi, \psi^{\prime}}: \mathcal{D}_{\alpha}^{p} \rightarrow L_{a}^{q, \alpha}$ is compact. So $\left\|W_{\varphi, \psi^{\prime}}\left(g_{n}\right)\right\|_{L_{a}^{q, \alpha}}^{q}$ also converges to zero. Now, we have

$$
\begin{aligned}
\left\|W_{\varphi, \psi \varphi^{\prime}}\left(f_{n}\right)\right\|_{L_{a}^{q, \alpha}}^{q} & =\left\|\psi \varphi^{\prime} f_{n} \circ \varphi\right\|_{L_{a}^{q, \alpha}}^{q} \\
& \leq\left\|\psi \varphi^{\prime} f_{n} \circ \varphi+\psi^{\prime} g_{n} \circ \varphi\right\|_{L_{a}^{q, \alpha}}+\left\|\psi^{\prime} g_{n} \circ \varphi\right\|_{L_{a}^{q, \alpha}}^{q} \\
& =\left\|\left(\psi g_{n} \circ \varphi\right)^{\prime}\right\|_{L_{a}^{q, \alpha}}^{q}+\left\|W_{\varphi, \psi^{\prime}}\left(g_{n}\right)\right\|_{L_{a}^{q, \alpha}}^{q} \\
& \leq\left\|W_{\varphi, \psi}\left(g_{n}\right)\right\|_{\mathcal{D}_{\alpha}^{q}}^{q}+\left\|W_{\varphi, \psi^{\prime}}\left(g_{n}\right)\right\|_{L_{a}^{q, \alpha}}^{q} .
\end{aligned}
$$


Therefore $\left\|W_{\varphi, \psi \varphi^{\prime}}\left(f_{n}\right)\right\|_{L^{q, \alpha}}^{q} \rightarrow 0$ as $n \rightarrow \infty$. Thus, $W_{\varphi, \psi \varphi^{\prime}}: L_{a}^{p, \alpha} \rightarrow L_{a}^{q, \alpha}$ is compact. Conversely, suppose $W_{\varphi, \psi \varphi^{\prime}}$ from $L_{a}^{p, \alpha}$ into $L_{a}^{q, \alpha}$ is compact. Then again, by Theorem 2.10, $W_{\varphi, \psi^{\prime}}: \mathcal{D}_{\alpha}^{p} \rightarrow L_{a}^{q, \alpha}$ is compact. Let $g_{n}$ be the same sequence as in the direct part. Then, we have

$$
\begin{aligned}
\left\|W_{\varphi, \psi}\left(g_{n}\right)\right\|_{\mathcal{D}_{\alpha}^{q}}^{q} & =\left\|\left(\psi g_{n} \circ \varphi\right)^{\prime}\right\|_{L_{a}^{q, \alpha}}^{q} \\
& =\left\|\psi \varphi^{\prime} g_{n}^{\prime} \circ \varphi+\psi^{\prime} g_{n} \circ \varphi\right\|_{L_{a}^{q, \alpha}}^{q} \\
& \leq\left\|W_{\varphi, \psi \varphi^{\prime}}\left(f_{n}\right)\right\|_{L_{a}^{q, \alpha}}^{q}+\left\|W_{\varphi, \psi^{\prime}}\left(g_{n}\right)\right\|_{L_{a}^{q, \alpha}}^{q} \rightarrow 0, \quad \text { as } n \rightarrow \infty .
\end{aligned}
$$

Thus, $W_{\varphi, \psi}: \mathcal{D}_{\alpha}^{p} \rightarrow \mathcal{D}_{\alpha}^{q}$ is compact. The proof is finished.

Theorem 2.15. Take $0<p<q<\infty$ and $-1<\alpha<\infty$. Suppose $\varphi \in \mathcal{D}_{\alpha}^{p}$ such that $\varphi$ is a self-map of $\mathbf{D}$ and let $\psi \in \mathcal{D}_{\alpha}^{q}$. Let $p<\alpha+2$ and suppose that the measure $\nu_{\varphi, \psi^{\prime}, q}$ is a $\frac{q}{p}(\alpha-p+2)$-Carleson measure. Then $W_{\varphi, \psi}$ from $\mathcal{D}_{\alpha}^{p}$ into $\mathcal{D}_{\alpha}^{q}$ is bounded if and only if the measure $\mu_{\varphi, \psi \varphi^{\prime}, q}$ is a $\frac{q}{p}(\alpha+2)$-Carleson measure for $L_{a}^{p, \alpha}$.

Proof. Suppose that $W_{\varphi, \psi}$ from $\mathcal{D}_{\alpha}^{p}$ into $\mathcal{D}_{\alpha}^{q}$ is bounded. Then, we can find a constant $C_{1}>0$ such that

$$
\left\|W_{\varphi, \psi}(f)\right\|_{\mathcal{D}_{\alpha}^{q}}^{q} \leq C_{1}\|f\|_{\mathcal{D}_{\alpha}^{p}}^{q}
$$

for all $f \in \mathcal{D}_{\alpha}^{p}$. Let $f \in \mathcal{D}_{\alpha}^{p}$ be such that $f(0)=0$. Also, by Theorem 2.11, we have $W_{\varphi, \psi \varphi^{\prime}}$ from $L_{a}^{p, \alpha}$ into $L_{a}^{q, \alpha}$ is bounded. Then there exists a constant $C_{2}>0$ such that

Thus

$$
\left\|W_{\varphi, \psi \varphi^{\prime}}\left(f^{\prime}\right)\right\|_{L_{a}^{q, \alpha}}^{q} \leq C_{2}\left\|f^{\prime}\right\|_{L_{a}^{p, \alpha}}^{q} \text { for all } f \in \mathcal{D}_{\alpha}^{p} .
$$

$$
\int_{\mathbf{D}}\left|\psi(z) \varphi^{\prime}(z)\right|^{q}\left|\left(f^{\prime} \circ \varphi\right)(z)\right|^{q} d A_{\alpha}(z) \leq C_{2}\left\|f^{\prime}\right\|_{L_{a}^{p, \alpha}}^{q},
$$

for all $f \in \mathcal{D}_{\alpha}^{p}$. Therefore by Lemma 2.2, we get

$$
\int_{\mathbf{D}}\left|f^{\prime}(w)\right|^{q} d \mu_{\varphi, \psi \varphi^{\prime}, q}(w) \leq C_{2}\left\|f^{\prime}\right\|_{L_{a}^{p, \alpha}}^{q} .
$$

Thus by Theorem 2.3, we get that $\mu_{\varphi, \psi \varphi^{\prime}, q}$ is a $\frac{q}{p}(\alpha+2)$-Carleson measure for $L_{a}^{p, \alpha}$.

Conversely, suppose that the measure $\mu_{\varphi, \psi \varphi^{\prime}, q}$ is a $\frac{q}{p}(\alpha+2)$-Carleson measure. Also, by hypothesis the measure $\nu_{\varphi, \psi^{\prime}, q}$ is a $\frac{q}{p}(\alpha-p+2)$-Carleson measure. Our aim is to prove that $W_{\varphi, \psi}: \mathcal{D}_{\alpha}^{p} \rightarrow \mathcal{D}_{\alpha}^{q}$ is bounded. Also, we have $(\psi(f \circ \varphi))^{\prime}=$ $\psi \varphi^{\prime}\left(f^{\prime} \circ \varphi\right)+\psi^{\prime}(f \circ \varphi)$. Take $f \in \mathcal{D}_{\alpha}^{p}$. So by Lemma 2.2, we have

$$
\int_{\mathbf{D}}\left|\psi(z) \varphi^{\prime}(z)\right|^{q}\left|f^{\prime}(\varphi(z))\right|^{q} d A_{\alpha}(z)=\int_{\mathbf{D}}\left|f^{\prime}(\omega)\right|^{q} d \mu_{\varphi, \psi \varphi^{\prime}, q}(\omega)<\infty .
$$

Again, since $f \in \mathcal{D}_{\alpha}^{p}$, we have

$$
\int_{\mathbf{D}}\left|\psi^{\prime}(z)\right|^{q}|f(\varphi(z))|^{q} d A_{\alpha}(z)=\int_{\mathbf{D}}|f(\omega)|^{q} d \nu_{\varphi, \psi^{\prime}, q}(\omega)<\infty .
$$

Thus $W_{\varphi, \psi}: \mathcal{D}_{\alpha}^{p} \rightarrow \mathcal{D}_{\alpha}^{q}$ is bounded. The proof is finished. 
Theorem 2.16. Let $1 \leq q<p<\infty$ and $-1<\alpha<\infty$. Let $\varphi$ be an analytic selfmap on the unit disk $\mathbf{D}$ and $\psi \in L_{a}^{q, \alpha}$. Then $W_{\varphi, \psi}$ is bounded from $L_{a}^{p, \alpha}$ into $L_{a}^{q, \alpha}$ if and only if $B_{\alpha}\left(\nu_{\varphi, \psi, q}\right) \in L_{a}^{p / p-q, \alpha}$.

Proof. We know that $W_{\varphi, \psi}$ is bounded from $L_{a}^{p, \alpha}$ into $L_{a}^{q, \alpha}$ if and only if for any $f \in L_{a}^{p, \alpha}$, there is a constant $C>0$ such that

$$
\left\|W_{\varphi, \psi}(f)\right\|_{L_{a}^{q, \alpha}}^{q} \leq C\|f\|_{L_{a}^{p, \alpha}}^{q} .
$$

This means that

$$
\int_{\mathbf{D}}|\psi(z)|^{q}|(f \circ \varphi)(z)|^{q} d A_{\alpha}(z) \leq C\|f\|_{L_{a}^{p, \alpha}}^{q} .
$$

Therefore from Lemma 2.2, we have

$$
\int_{\mathbf{D}}|f(w)|^{q} d \nu_{\varphi, \psi^{\prime}, q}(\omega) \leq C\|f\|_{L_{a}^{p, \alpha}}^{q} .
$$

Thus $\nu_{\varphi, \psi^{\prime}, q}$ is a $\left(L_{a}^{p, \alpha}, q\right)$-Carleson measure and so $B_{\alpha}\left(\nu_{\varphi, \psi, q}\right) \in L_{a}^{p / p-q, \alpha}$.

Theorem 2.17. Suppose that $0<p<q<\infty$ and $-1<\alpha<\infty$. Let $\varphi \in \mathcal{D}_{\alpha}^{p}$ be such that $\varphi$ is a self-map of $\mathbf{D}$ and let $\psi \in \mathcal{D}_{\alpha}^{q}$. For $p<\alpha+2$, suppose that the measure $\nu_{\varphi, \psi^{\prime}, q}$ is a vanishing $\frac{q}{p}(\alpha-p+2)$-Carleson measure. Then $W_{\varphi, \psi}$ from $\mathcal{D}_{\alpha}^{p}$ into $\mathcal{D}_{\alpha}^{q}$ is compact if and only if the measure $\mu_{\varphi, \psi \varphi^{\prime}, q}$ is a vanishing $\frac{q}{p}(\alpha+2)$-Carleson measure for $L_{a}^{p, \alpha}$.

Proof. Suppose that the measure $\mu_{\varphi, \psi \varphi^{\prime}, q}$ is a vanishing $\frac{q}{p}(\alpha+2)$-Carleson measure for $L_{a}^{p, \alpha}$. Also, by hypothesis the measure $\nu_{\varphi, \psi^{\prime}, q}$ is a vanishing $\frac{q}{p}(\alpha-p+2)$-Carleson measure. We show that the operator $W_{\varphi, \psi}$ from $\mathcal{D}_{\alpha}^{p}$ into $\mathcal{D}_{\alpha}^{q}$ is compact. Let $f_{n}$ be a bounded sequence in $\mathcal{D}_{\alpha}^{p}$ with $f_{n} \rightarrow 0$ uniformly on compact subsets of $\mathbf{D}$, we have to prove that $\left\|W_{\varphi, \psi}\left(f_{n}\right)\right\|_{\mathcal{D}_{\alpha}^{q}}^{q} \rightarrow 0$. Again, since $\left\|W_{\varphi, \psi}\left(f_{n}\right)\right\|_{\mathcal{D}_{\alpha}^{q}}^{q}=\left|\psi\left(f_{n} \circ \varphi\right)(0)\right|+$ $\left\|\left(\psi\left(f_{n} \circ \varphi\right)\right)^{\prime}\right\|_{L_{a}^{q, \alpha}}^{q}$, we only have to show that $\left\|\left(\psi\left(f_{n} \circ \varphi\right)\right)^{\prime}\right\|_{L_{a}^{q, \alpha}}^{q} \rightarrow 0$. Again by Theorem 2.10, we have $\psi\left(f_{n} \circ \varphi\right) \in \mathcal{D}_{\alpha}^{p}$ for every $n$. Hence

$$
\begin{aligned}
\left\|\left(\psi\left(f_{n} \circ \varphi\right)\right)^{\prime}\right\|_{L_{a}^{q, \alpha}}^{q} \leq & \int_{\mathbf{D}}\left|\psi(z) \varphi^{\prime}(z)\right|^{q}\left|\left(f_{n}^{\prime} \circ \varphi\right)(z)\right|^{q} d A_{\alpha}(z) \\
& +\int_{\mathbf{D}}\left|\left(\psi^{\prime}\left(f_{n} \circ \varphi\right)\right)(z)\right|^{q} d A_{\alpha}(z) \\
= & \int_{\mathbf{D}}\left|f_{n}^{\prime}(w)\right|^{q} d \mu_{\varphi, \psi \varphi^{\prime}, q}(w)+\int_{\mathbf{D}}\left|f_{n}(w)\right|^{q} d \nu_{\varphi, \psi^{\prime}, q}(w) .
\end{aligned}
$$

We replace the sets $S(b, h)$ by the equivalent windows $W(b, h)$ defined as

$$
W(b, h)=\left\{z \in \overline{\mathbf{D}}, 1-|z|<h, \frac{z}{|z|} \in Q(b, h)\right\},
$$


where $Q(b, h)=S(b, h) \cap \partial \mathbf{D}$. Given $\epsilon>0$, we may find $h_{\circ}$ so that $\mu(W(b, h)) \leq$ $\epsilon h^{\frac{q}{p}(\alpha+2)}$ for all $b \in \partial \mathbf{D}$ and $h \leq h_{\circ}$. If $\mu$ is the restriction of $\mu_{q}$ to $\mathbf{D} \backslash\left(1-h_{\circ}\right) \mathbf{D}$, then $\mu$ is a $\frac{q}{p}(\alpha+2)$-Carleson measure with $\mu(W(b, h)) \leq 2 \epsilon h^{\frac{q}{p}(\alpha+2)}$ (see [3, Theorem 3.12]). This implies that there is a constant $C<\infty$ such that

$$
\int_{\mathbf{D}}|f(w)|^{q} d \mu(w) \leq C \epsilon\|f\|_{L_{a}^{p, \alpha}}^{q},
$$

for all $f \in L_{a}^{p, \alpha}$. Now, take the integral

$$
\begin{aligned}
\int_{\mathbf{D}}\left|f_{n}^{\prime}(w)\right|^{q} d \mu_{\varphi, \psi \varphi^{\prime}, q}(w)= & \int_{\mathbf{D} \backslash\left(1-h_{\circ}\right) \mathbf{D}}\left|f_{n}^{\prime}(w)\right|^{q} d \mu_{\varphi, \psi \varphi^{\prime}, q}(w) \\
& +\int_{\left(1-h_{\circ}\right) \mathbf{D}}\left|f_{n}^{\prime}(w)\right|^{q} d \mu_{\varphi, \psi \varphi^{\prime}, q}(w) .
\end{aligned}
$$

Since $f_{n} \rightarrow 0$ uniformly on compact subsets of $\mathbf{D}$, we can find $N_{\circ}$ such that if $n \in \mathbf{N}$ and $n \geq N_{\circ}$, we have that $\left|f_{n}^{\prime}(z)\right|^{q} \leq \frac{\epsilon}{\mu_{\varphi, \psi \varphi^{\prime}, q}\left(\left(1-h_{\circ}\right) \mathbf{D}\right)}$, for all $z \in\left(1-h_{\circ}\right) \mathbf{D}$. Thus we have

$$
\int_{\left(1-h_{\circ}\right) \mathbf{D}}\left|f_{n}^{\prime}(w)\right|^{q} d \mu_{\varphi, \psi \varphi^{\prime}, q}(w) \leq \frac{\epsilon}{\mu_{\varphi, \psi \varphi^{\prime}, q}\left(\left(1-h_{\circ}\right) \mathbf{D}\right)} \mu_{\varphi, \psi \varphi^{\prime}, q}\left(\left(1-h_{\circ}\right) \mathbf{D}\right)=\epsilon .
$$

Also

$$
\begin{aligned}
\int_{\mathbf{D} \backslash\left(1-h_{\circ}\right) \mathbf{D}}\left|f_{n}^{\prime}(w)\right|^{q} d \mu_{\varphi, \psi \varphi^{\prime}, q}(w) & =\int_{\mathbf{D} \backslash\left(1-h_{\circ}\right) \mathbf{D}}\left|f_{n}^{\prime}(w)\right|^{q} d \mu(w) \\
& \leq \int_{\mathbf{D}}\left|f_{n}^{\prime}(w)\right|^{p} d \mu(w) \\
& \leq \epsilon C\left\|f_{n}^{\prime}\right\|_{L_{a}^{p, \alpha}} \\
& \leq \epsilon C,
\end{aligned}
$$

because we can take $\left\|f_{n}^{\prime}\right\|_{L_{a}^{q, \alpha}}^{q} \leq 1$. Thus we get

$$
\int_{\mathbf{D}}\left|f_{n}^{\prime}(w)\right|^{q} d \mu_{\varphi, \psi \varphi^{\prime}, q}(w) \leq(1+C) \epsilon .
$$

Since the measure $\nu_{\varphi, \psi^{\prime}, q}$ is a vanishing $\frac{q}{p}(\alpha-p+2)$-Carleson measure, we have $\int_{\mathbf{D}}\left|f_{n}(w)\right|^{q} d \nu_{\varphi, \psi^{\prime}, q}(w) \rightarrow 0$ as $n \rightarrow \infty$. Thus $W_{\varphi, \psi}$ from $\mathcal{D}_{\alpha}^{p}$ into $\mathcal{D}_{\alpha}^{q}$ is compact.

Conversely, suppose that $W_{\varphi, \psi}$ is compact. Our aim is to prove that the measure $\mu_{\varphi, \psi \varphi^{\prime}, q}$ is a vanishing $\frac{q}{p}(\alpha+2)$-Carleson measure for $L_{a}^{p, \alpha}$. By Theorem 2.10, we have $W_{\varphi, \psi^{\prime}}$ from $\mathcal{D}_{\alpha}^{p}$ into $L_{a}^{q, \alpha}$ is compact. Let $\left\{f_{n}\right\}$ be a bounded sequence in $L_{a}^{q, \alpha}$ such that $f_{n} \rightarrow 0$ uniformly on compact subsets of $\mathbf{D}$. Consider the function $g_{n} \in \mathcal{D}_{\alpha}^{p}$ such that $g_{n}^{\prime}=f_{n}$ and $g_{n}(0)=0$, for each $n$. Then the sequence $g_{n}$ also converges to 
zero uniformly on compact subsets of $\mathbf{D}$ as $n \rightarrow \infty$. Also by Theorem 2.14, $W_{\varphi, \psi \varphi^{\prime}}$ : $L_{a}^{p, \alpha} \rightarrow L_{a}^{q, \alpha}$ is compact. So $\left\|W_{\varphi, \psi \varphi^{\prime}}\left(f_{n}\right)\right\|_{L_{a}^{q, \alpha}} \rightarrow 0$. Thus, we have

$$
\begin{aligned}
\left\|W_{\varphi, \psi \varphi^{\prime}}\left(f_{n}\right)\right\|_{L_{\alpha}^{q, \alpha}}^{q} & =\int_{\mathbf{D}}\left|\psi(z) \varphi^{\prime}(z)\right|^{p}\left|\left(f_{n} \circ \varphi\right)(z)\right|^{q} d A_{\alpha}(z) \\
& =\int_{\mathbf{D}}\left|f_{n}(w)\right|^{q} d \mu_{\varphi, \psi \varphi^{\prime}, q}(w) \rightarrow 0, \quad \text { as } n \rightarrow \infty .
\end{aligned}
$$

Therefore by Theorem 2.15, we see that $\mu_{\varphi, \psi \varphi^{\prime}, q}$ is a vanishing $\frac{q}{p}(\alpha+2)$-Carleson measure for $L_{a}^{p, \alpha}$. The proof is finished.

Theorem 2.18. Suppose that $0<q<p \leq 2, \alpha=p-1$ and the measure $\nu_{\varphi, \psi^{\prime}, q}$ is a vanishing 1-Carleson measure for $\mathcal{D}_{\alpha}^{p}$. Also suppose that $W_{\varphi, \psi}$ is bounded (respectively compact) on $\mathcal{D}_{\alpha}^{p}$. Then $W_{\varphi, \psi}$ is bounded (respectively compact) on $\mathcal{D}_{\alpha}^{q}$.

Proof. For $q<p$, by Hölders inequality we have

$$
\begin{aligned}
\mu_{\varphi, \psi \varphi^{\prime}, q}(S(b, h)) & =\int_{\varphi^{-1}(S(b, h))}\left|\psi(z) \varphi^{\prime}(z)\right|^{q} d A_{\alpha}(z) \\
& \leq\left(\int_{\varphi^{-1}(S(b, h))}\left|\psi(z) \varphi^{\prime}(z)\right|^{p} d A_{\alpha}(z)\right)^{\frac{q}{p}}\left(\int_{\varphi^{-1}(S(b, h))} 1 d A_{\alpha}(z)\right)^{\frac{(p-q)}{p}} \\
& =\mu_{\varphi, \psi \varphi^{\prime}, p}(S(b, h))^{\frac{q}{p}} A_{\alpha} \varphi^{-1}(S(b, h))^{\frac{(p-q)}{p}} .
\end{aligned}
$$

Also, the composition operator $C_{\varphi}$ is bounded on the Bergman space, so by Theorem 4.3 in [14], $A_{\alpha} \varphi^{-1}$ is a Carleson measure for the Bergman space. Thus

$$
\mu_{\varphi, \psi \varphi^{\prime}, q}(S(b, h)) \leq C h^{\frac{(p-q)}{p}} \mu_{\varphi, \psi \varphi^{\prime}, p}(S(b, h))^{\frac{q}{p}} .
$$

Again $W_{\varphi, \psi}$ is bounded on $\mathcal{D}_{\alpha}^{p}$, by Theorem 2.15, we can find a constant $C$ such that $\mu_{\varphi, \psi \varphi^{\prime}, p}(S(b, h)) \leq C h^{(\alpha+2)}$ for all $b \in \partial \mathbf{D}$ and $0<h \leq 2$. Thus we have

$$
\mu_{\varphi, \psi \varphi^{\prime}, q}(S(b, h)) \leq C h^{(\alpha+2)} .
$$

That is, the measure $\mu_{\varphi, \psi \varphi^{\prime}, q}$ is a $(\alpha+2)$-Carleson measure for $L_{a}^{p, \alpha}$. Hence by Theorem $2.15, W_{\varphi, \psi}$ is bounded on $\mathcal{D}_{\alpha}^{q}$.

Similarly, we can prove this result for compactness.

Our next result concerns bounded weighted composition operators mapping $\mathcal{D}_{\alpha}^{p}$ into $\mathcal{D}_{\alpha}^{q}$ for $0<p<q<\infty, p<\alpha+2$ and $-1<\alpha<\infty$. Our result will be expressed in term of intergal operators:

$$
\Phi(a)=\int_{\mathbf{D}}\left(\frac{1-|a|^{2}}{|1-\bar{a} z|^{2}}\right)^{\frac{q}{p}(\alpha+2)} d \mu_{\varphi, \psi \varphi^{\prime}, q}(\omega)
$$


and

$$
\Psi(a)=\int_{\mathbf{D}}\left(\frac{1-|a|^{2}}{|1-\bar{a} z|^{2}}\right)^{\frac{q}{p}(\alpha-p+2)} d \nu_{\varphi, \psi^{\prime}, q}(\omega) .
$$

Theorem 2.19. Let $\varphi \in \mathcal{D}_{\alpha}^{p}$ be such that $\varphi(\mathbf{D}) \subseteq \mathbf{D}$ and $\psi \in \mathcal{D}_{\alpha}^{q}$. Let $0<p<q<\infty$ and $-1<\alpha<\infty$. For $p<\alpha+2$, suppose that the measure $\nu_{\varphi, \psi^{\prime}, q}$ is a $\frac{q}{p}(\alpha-p+2)$ Carleson measure. Then the weighted composition operator $W_{\varphi, \psi}$ from $\mathcal{D}_{\alpha}^{p}$ into $\mathcal{D}_{\alpha}^{q}$ is bounded if and only if the functions $\Phi(a)$ and $\Psi(a)$ belong to $L^{\infty}(\mathbf{D})$.

Proof. Since $W_{\varphi, \psi}: \mathcal{D}_{\alpha}^{p} \rightarrow \mathcal{D}_{\alpha}^{q}$ is bounded, by using Theorem 2.15, we get that the measure $\mu_{\varphi, \psi \varphi^{\prime}, q}$ is a $\frac{q}{p}(\alpha+2)$ Carleson measure for $L_{a}^{p, \alpha}$. Therefore by Theorem A of $[5]$, we have

$$
\int_{\mathbf{D}}\left|\sigma_{a}^{\prime}(w)\right|^{\frac{q}{p}(\alpha+2)} d \mu_{\varphi, \psi \varphi^{\prime}, q}(w)<\infty
$$

That is,

$$
\int_{\mathbf{D}}\left(\frac{1-|a|^{2}}{|1-\bar{a} z|^{2}}\right)^{\frac{q}{p}(\alpha+2)} d \mu_{\varphi, \psi \varphi^{\prime}, q}(\omega)<\infty .
$$

Again by hypothesis, the measure $\nu_{\varphi, \psi^{\prime}, q}$ is a $\frac{q}{p}(\alpha-p+2)$ Carleson measure. Thus by Theorem 2.7 , we have

$$
\int_{\mathbf{D}}\left|\sigma_{a}^{\prime}(w)\right|^{\frac{q}{p}(\alpha-p+2)} d \nu_{\varphi, \psi^{\prime}, q}(w)<\infty
$$

That is,

$$
\int_{\mathbf{D}}\left(\frac{1-|a|^{2}}{|1-\bar{a} z|^{2}}\right)^{\frac{q}{p}(\alpha-p+2)} d \nu_{\varphi, \psi^{\prime}, q}(\omega)<\infty
$$

\section{Essential norm estimates}

In this section, we find the estimates for the essential norm of $W_{\varphi, \psi}$.

We need the following two lemmas.

Lemma 3.1. For $0<r<1$, let us denote $\mathbf{D}_{r}=\{z \in \mathbf{D}:|z|<r\}$. Let $\mu$ be a positive Borel measure on D. Take

$$
\|\mu\|_{r}=\sup _{|I| \leq 1-r} \frac{\mu(S(I))}{|I|^{\frac{q}{p}(\alpha-p+2)}} \quad \text { and } \quad\|\mu\|=\sup _{I \subset \partial \mathbf{D}} \frac{\mu(S(I))}{|I|^{\frac{q}{p}(\alpha-p+2)}},
$$

where $I$ is an arc in the unit circle $\partial \mathbf{D}$. Let $\mu_{r}=\mathbf{D} \backslash \mathbf{D}_{r}$. Thus, if $\mu$ is a Carleson measure for the weighted Dirichlet spaces $\mathcal{D}_{\alpha}^{p}$ for $0<p<q<\infty, p<\alpha+1$ and $-1<\alpha<\infty$, then so is $\mu_{r}$. Also $\left\|\mu_{r}\right\| \leq M\|\mu\|_{r}$, where $M>0$ is a constant. 
Lemma 3.2. For $0<r<1$, let $\mu$ be a positive Borel measure on D. Denote

$$
\|\mu\|_{r}^{*}=\sup _{|a| \geq r} \int_{\mathbf{D}}\left|\sigma_{a}^{\prime}(z)\right|^{\frac{q}{p}(\alpha-p+2)} d \mu(z) .
$$

If $\mu$ is a $\left(\mathcal{D}_{\alpha}^{p}, q\right)$-Carleson measure for $0<p<q<\infty, p<\alpha+2$ and $-1<\alpha<\infty$, then so is $\mu_{r}$. Also, $\left\|\mu_{r}\right\| \leq C\|\mu\|_{r}^{*}$, where $C$ is an absolute constant.

Proof. By Lemma 3.1, we only need to show that $\|\mu\|_{r} \leq C\|\mu\|_{r}^{*}$, where $C$ is an absolute constant. Take an arc $I \subset \partial \mathbf{D}$ with $|I| \leq 1-r$. Let $a=(1-|I|) e^{i \theta}$, where $e^{i \theta}$ is the centre of $I$. Then $|a|=(1-|I|) \geq r$. By a geometric consideration, it can be proved that for any $z \in S(I),\left|\sigma_{a}^{\prime}(z)\right| \geq 4 /(25|I|)$. Thus

$$
\begin{aligned}
\frac{\mu(S(I))}{|I|^{\frac{q}{p}(\alpha-p+2)}} & \leq(25 / 4)^{\frac{q}{p}(\alpha-p+2)} \int_{S(I)}\left|\sigma_{a}^{\prime}(z)\right|^{\frac{q}{p}(\alpha-p+2)} d \mu(z) \\
& \leq(25 / 4)^{\frac{q}{p}(\alpha-p+2)} \int_{\mathbf{D}}\left|\sigma_{a}^{\prime}(z)\right|^{\frac{q}{p}(\alpha-p+2)} d \mu(z) \\
& \leq(25 / 4)^{\frac{q}{p}(\alpha-p+2)}\|\mu\|_{r}^{*} .
\end{aligned}
$$

Taking supremum over the $\operatorname{arcs} I$ with $|I| \leq 1-r$, we get

$$
\|\mu\|_{r} \leq(25 / 4)^{\frac{q}{p}(\alpha-p+2)}\|\mu\|_{r}^{*} .
$$

Therefore by Lemma 3.1, $\left\|\mu_{r}\right\| \leq M\left\|\mu_{r}\right\| \leq C\|\mu\|_{r}^{*}$. Thus the result is proved with $C=M(25 / 4)^{\frac{q}{p}(\alpha-p+2)}$.

Lemma 3.1 and Lemma 3.2 can also be proved for the weighted Bergman spaces. Only we have to take exponent $\frac{q}{p}(\alpha+2)$ in place of $\frac{q}{p}(\alpha-p+2)$.

Take $f(z)=\sum_{t=0}^{\infty} a_{t} z^{t}$ analytic in $\mathbf{D}$. For a positive integer $\mathrm{n}$, define the operators $R_{n} f(z)=\sum_{t=n+1}^{\infty} a_{t} z^{t}$ and $K_{n}=I-R_{n}$, where $I$ is the identity map.

Now recall that the essential norm $\left\|W_{\varphi, \psi}\right\|_{e}$ of a bounded operator $W_{\varphi, \psi}$ is its distance (in the operator norm) from compact operators, that is,

$$
\left\|W_{\varphi, \psi}\right\|_{e}=\inf _{K}\left\|W_{\varphi, \psi}-K\right\|
$$

where the infimum is taken over all compact operators $K$.

Then we have the following lemma.

Lemma 3.3. If $W_{\varphi, \psi}$ is a bounded from $\mathcal{D}_{\alpha}^{p}$ into $\mathcal{D}_{\alpha}^{q}$ for $0<p<q<\infty, p<\alpha+2$ and $-1<\alpha<\infty$, then

$$
\left\|W_{\varphi, \psi}\right\|_{e} \leq \lim _{n \rightarrow \infty} \inf \left\|W_{\varphi, \psi} R_{n}\right\|
$$


Proof. Since $\left(R_{n}+K_{n}\right) f=f$ for every $n$, where $K_{n}$ is a compact operator. Then we have

$$
\left\|W_{\varphi, \psi} f\right\|=\left\|W_{\varphi, \psi}\left(R_{n}\right) f+W_{\varphi, \psi}\left(K_{n}\right) f\right\| .
$$

Thus

$$
\begin{aligned}
\left\|W_{\varphi, \psi}\right\|_{e} & \leq\left\|W_{\varphi, \psi} R_{n}+W_{\varphi, \psi} K_{n}\right\|_{e} \\
& \leq\left\|W_{\varphi, \psi} R_{n}\right\|_{e} \\
& \leq\left\|W_{\varphi, \psi} R_{n}\right\| .
\end{aligned}
$$

Therefore $\left\|W_{\varphi, \psi}\right\|_{e} \leq \lim _{n \rightarrow \infty} \inf \left\|W_{\varphi, \psi} R_{n}\right\|$.

In the following theorem, we give the upper and lower estimates for the essential norm of a weighted composition operator.

Theorem 3.4. Let $\varphi \in \mathcal{D}_{\alpha}^{p}$ be such that $\varphi(\mathbf{D}) \subseteq \mathbf{D}$ and $\psi \in \mathcal{D}_{\alpha}^{q}$. Take $0<p<q<\infty$ and $-1<\alpha<\infty$. For $p<\alpha+2$, suppose that the measure $\nu_{\varphi, \psi^{\prime}, q}$ is a $\frac{q}{p}(\alpha-p+2)$ Carleson measure. Let $W_{\varphi, \psi}$ be bounded from $\mathcal{D}_{\alpha}^{p}$ into $\mathcal{D}_{\alpha}^{q}$. Then there are absolute constants $C_{1}, C_{2} \geq 1$ such that

$$
\limsup _{|a| \rightarrow 1}\left\|\left(W_{\varphi, \psi}\right) \sigma_{a}\right\|_{\mathcal{D}_{\alpha}^{q}}^{q} \leq\left\|W_{\varphi, \psi}\right\|_{e}^{q} \leq C_{1} \limsup _{|a| \rightarrow 1} \Phi(a)+C_{2} \limsup _{|a| \rightarrow 1} \Psi(a),
$$

where

$$
\Phi(a)=\int_{\mathbf{D}}\left(\frac{1-|a|^{2}}{|1-\bar{a} z|^{2}}\right)^{\frac{q}{p}(\alpha+2)} d \mu_{\varphi, \psi \varphi^{\prime}, q}(\omega)
$$

and

$$
\Psi(a)=\int_{\mathbf{D}}\left(\frac{1-|a|^{2}}{|1-\bar{a} z|^{2}}\right)^{\frac{q}{p}(\alpha-p+2)} d \nu_{\varphi, \psi^{\prime}, q}(\omega)
$$

Proof. First we prove the upper estimate. Now by Lemma 3.3, we have

$$
\begin{aligned}
\left\|W_{\varphi, \psi}\right\|_{e}^{q} & \leq \lim _{n \rightarrow \infty} \inf \left\|W_{\varphi, \psi} R_{n}\right\|_{\mathcal{D}_{\alpha}^{q}}^{q} \\
& \leq \lim _{n \rightarrow \infty} \inf \sup _{\|f\|_{\mathcal{D}_{\alpha}^{p} \leq 1}\left\|\left(W_{\varphi, \psi} R_{n}\right) f\right\|_{\mathcal{D}_{\alpha}^{q}}^{q} .}
\end{aligned}
$$

However, for any fixed $0<r<1$,

$$
\begin{aligned}
\left\|W_{\varphi, \psi} R_{n} f\right\|_{\mathcal{D}_{\alpha}^{q}}^{q} & =\mid \psi(0)\left(\left.R_{n} f(\varphi(0))\right|^{q}+\left\|\left(\psi(2)\left(R_{n} f \circ \varphi\right)(2)\right)^{\prime}\right\|_{L_{\alpha}^{p, \alpha}}^{q}\right. \\
& =\mid \psi(0)\left(\left.R_{n} f(\varphi(0))\right|^{q}+\int_{\mathbf{D}}\left|\left(\psi(z) R_{n} f(\varphi(z))\right)^{\prime}\right|^{q} d A_{\alpha}(z) .\right.
\end{aligned}
$$


Now the term $\mid \psi(0)\left(R_{n} f(\varphi(0)) \mid\right.$ is bounded as $n \rightarrow \infty$. Thus we have

$$
\begin{aligned}
\left\|\left(W_{\varphi, \psi} R_{n}\right) f\right\|_{\mathcal{D}_{\alpha}^{q}}^{q}= & \int_{\mathbf{D}}\left|\left(\psi(z)\left(R_{n} f(\varphi(z))\right)\right)^{\prime}\right|^{q} d A_{\alpha}(z) \\
\leq & \int_{\mathbf{D}}\left|\psi(z) \varphi^{\prime}(z)\right|^{q}\left|\left(R_{n} f\right)^{\prime}(\varphi(z))\right|^{q} d A_{\alpha}(z) \\
& +\int_{\mathbf{D}}\left|\psi^{\prime}(z)\right|^{q}\left|\left(R_{n} f\right)(\varphi(z))\right|^{q} d A_{\alpha}(z) . \\
= & \int_{\mathbf{D}}\left|\left(R_{n} f\right)^{\prime}(\omega)\right|^{q} d \mu_{\varphi, \psi \varphi^{\prime}, q}(\omega) \\
& +\int_{\mathbf{D}}\left|\left(R_{n} f\right)(\omega)\right|^{q} d \nu_{\varphi, \psi^{\prime}, q}(\omega) . \\
= & \int_{\mathbf{D} \backslash \mathbf{D}_{r}}\left|\left(R_{n} f\right)^{\prime}(\omega)\right|^{q} d \mu_{\varphi, \psi \varphi^{\prime}, q}(\omega) \\
& +\int_{\mathbf{D}_{r}}\left|\left(R_{n} f\right)^{\prime}(\omega)\right|^{q} d \mu_{\varphi, \psi \varphi^{\prime}, q}(\omega) \\
& +\int_{\mathbf{D}}\left|\left(R_{n} f\right)(\omega)\right|^{q} d \nu_{\varphi, \psi^{\prime}, q}(\omega) \\
= & I_{1}+I_{2}+I_{3} .
\end{aligned}
$$

Also by Theorem 2.15, $W_{\varphi, \psi}$ from $\mathcal{D}_{\alpha}^{p}$ into $\mathcal{D}_{\alpha}^{q}$ is bounded. So the measure $\mu_{\varphi, \psi \varphi^{\prime}, q}$ is a $\frac{q}{p}(\alpha+2)$-Carleson measure. From the proof of Proposition 3 in [4], we see that for a given $\epsilon>0$ and $n$ large enough,

$$
\left|\left(R_{n} f\right)^{\prime}(\omega)\right| \leq \epsilon\left\|f^{\prime}\right\|_{L_{a}^{p, \alpha} .}
$$

Thus

$$
I_{2} \leq \epsilon^{q}\|f\|_{\mathcal{D}_{\alpha}^{p}}^{q} \mu_{\varphi, \psi \varphi^{\prime}, q}\left(D_{r}\right) \leq \epsilon^{q}\|f\|_{\mathcal{D}_{\alpha}^{p}}^{q}\left\|\psi \varphi^{\prime}\right\|_{L_{a}^{q, \alpha}} .
$$

Therefore for a fixed $r$, we have

$$
\sup _{\|f\|_{\mathcal{D}_{\alpha}^{q} \leq 1}} \int_{\mathbf{D}_{r}}\left|\left(R_{n} f\right)^{\prime}(\omega)\right|^{q} d \mu_{\varphi, \psi \varphi^{\prime}, q}(\omega) \rightarrow 0, \quad \text { as } n \rightarrow \infty .
$$

On the other hand, if $\mu_{\varphi, \psi \varphi^{\prime}, q, r}$ denotes the restriction of measure $\mu_{\varphi, \psi \varphi^{\prime}, q}$ to the set $\mathbf{D} \backslash \mathbf{D}_{r}$, then by Theorem 2.7 and Lemma 3.2, we have

$$
\begin{aligned}
I_{1} & =\int_{\mathbf{D} \backslash \mathbf{D}_{r}}\left|\left(R_{n} f\right)^{\prime}(\omega)\right|^{q} d \mu_{\varphi, \psi \varphi^{\prime}, q, r}(\omega) \\
& \leq M\left\|\mu_{\varphi, \psi \varphi^{\prime}, q, r}\right\|\left\|\left(R_{n} f\right)^{\prime}\right\|_{L_{a}^{p, \alpha}}^{q} \\
& \leq M M^{\prime}\left\|\mu_{\varphi, \psi \varphi^{\prime}, q}\right\|_{r}^{*}\|f\|_{\mathcal{D}_{\alpha}^{p}}^{p} \\
& \leq M M^{\prime}\left\|\mu_{\varphi, \psi \varphi^{\prime}, q}\right\|_{r}^{*},
\end{aligned}
$$


where $M$ and $M^{\prime}$ are absolute constants and $\left\|\mu_{\varphi, \psi \varphi^{\prime}, q}\right\|_{r}^{*}$ is defined as in Lemma 3.2.

Also by using similar techniques as above, we can show that the intergal $I_{3}$ is also bounded by $M_{1} M_{1}^{\prime}\left\|\nu_{\varphi, \psi^{\prime}, q}\right\|_{r}^{*}$, where $M_{1}$ and $M_{1}^{\prime}$ are absolute constants. Therefore

$$
\begin{aligned}
\lim _{n \rightarrow \infty} \inf \sup _{\|f\|_{\mathcal{D}_{\alpha}^{p} \leq 1}\left\|\left(W_{\varphi, \psi} R_{n}\right) f\right\|_{\mathcal{D}_{\alpha}^{q}} \leq} & \lim _{n \rightarrow \infty} \inf M M^{\prime}\left\|\mu_{\varphi, \psi \varphi^{\prime}, q}\right\|_{r}^{*} \\
& +\lim _{n \rightarrow \infty} \inf M_{1} M_{1}^{\prime}\left\|\nu_{\varphi, \psi^{\prime}, q}\right\|_{r}^{*} .
\end{aligned}
$$

Thus $\left\|W_{\varphi, \psi}\right\|_{e}^{q} \leq M M^{\prime}\left\|\mu_{\varphi, \psi \varphi^{\prime}, q}\right\|_{r}^{*}+M_{1} M_{1}^{\prime}\left\|\nu_{\varphi, \psi^{\prime}, q}\right\|_{r}^{*}$.

Taking $r \rightarrow 1$, we have

$$
\begin{aligned}
\left\|W_{\varphi, \psi}\right\|_{e}^{q} \leq & M M^{\prime} \lim _{r \rightarrow 1}\left\|\mu_{\varphi, \psi \varphi^{\prime}, q}\right\|_{r}^{*}+M_{1} M_{1}^{\prime} \lim _{r \rightarrow 1}\left\|\nu_{\varphi, \psi^{\prime}, q}\right\|_{r}^{*} \\
= & M M^{\prime} \limsup _{|a| \rightarrow 1} \int_{\mathbf{D}}\left|\sigma_{a}^{\prime}(\omega)\right|^{(\alpha+2) q / p} d \mu_{\varphi, \psi \varphi^{\prime}, q}(\omega) \\
& +M_{1} M_{1}^{\prime} \limsup _{|a| \rightarrow 1} \int_{\mathbf{D}}\left|\sigma_{a}^{\prime}(\omega)\right|^{\frac{q}{p}(\alpha-p+2)} d \nu_{\varphi, \psi^{\prime}, q}(\omega) \\
= & M M^{\prime} \limsup _{|a| \rightarrow 1} \int_{\mathbf{D}}\left(\frac{1-|a|^{2}}{|1-\bar{a} \omega|^{2}}\right)^{(\alpha+2) q / p} d \mu_{\varphi, \psi \varphi^{\prime}, q}(\omega) \\
& +M_{1} M_{1}^{\prime} \limsup _{|a| \rightarrow 1} \int_{\mathbf{D}}\left(\frac{1-|a|^{2}}{|1-\bar{a} \omega|)^{2}}\right)^{\frac{q}{p}(\alpha-p+2)} d \nu_{\varphi, \psi^{\prime}, q}(\omega) \\
= & M M^{\prime} \limsup _{|a| \rightarrow 1} \Phi(a)+M_{1} M_{1}^{\prime} \limsup _{|a| \rightarrow 1} \Psi(a),
\end{aligned}
$$

which is the desired upper bound.

Now, we prove the lower estimate.

The set $\left\{\sigma_{a}: a \in \mathbf{D}\right\}$ is bounded in $\mathcal{D}_{\alpha}^{p}$. Also $\left(\sigma_{a}-a\right) \rightarrow 0$ as $|a| \rightarrow 1$ uniformly on compact sets in $\mathbf{D}$, since

$$
\left|\sigma_{a}(z)-a\right|=|z| \frac{1-|a|^{2}}{|1-\bar{a} z|} .
$$

Also, fix a compact operator $K$ from $\mathcal{D}_{\alpha}^{p}$ into $\mathcal{D}_{\alpha}^{q}$. Then $\left\|K\left(\sigma_{a}-a\right)\right\|_{\mathcal{D}_{\alpha}^{q}} \rightarrow 0$, as $|a| \rightarrow 1$. Thus $\left\|K\left(\sigma_{a}\right)\right\|_{\mathcal{D}_{\alpha}^{q}} \rightarrow 0$, as $|a| \rightarrow 1$. Therefore

$$
\begin{aligned}
\left\|W_{\varphi, \psi}-K\right\| & \geq \lim _{|a| \rightarrow 1} \sup \left\|\left(W_{\varphi, \psi}-K\right) \sigma_{a}\right\|_{\mathcal{D}_{\alpha}^{q}} \\
& \geq \lim _{|a| \rightarrow 1} \sup \left(\left\|\left(W_{\varphi, \psi}\right) \sigma_{a}\right\|_{\mathcal{D}_{\alpha}^{q}}-\left\|K \sigma_{a}\right\|_{\mathcal{D}_{\alpha}^{q}}\right. \\
& =\lim _{|a| \rightarrow 1} \sup \left\|\left(W_{\varphi, \psi} \sigma_{a}\right)\right\|_{\mathcal{D}_{\alpha}^{q}} .
\end{aligned}
$$

Hence

$$
\left\|W_{\varphi, \psi}\right\|_{e}^{q} \geq\left\|W_{\varphi, \psi}-K\right\|_{\mathcal{D}_{\alpha}^{q}}^{q} \geq \limsup _{|a| \rightarrow 1}\left\|\left(W_{\varphi, \psi}\right) \sigma_{a}\right\|_{\mathcal{D}_{\alpha}^{q}}^{q} .
$$


Corollary 3.5. Let $\varphi \in \mathcal{D}_{\alpha}^{p}$ be such that $\varphi(\mathbf{D}) \subseteq \mathbf{D}$ and let $\psi \in \mathcal{D}_{\alpha}^{q}$. Take $0<$ $p<q<\infty$ and $-1<\alpha<\infty$. For $p<\alpha+2$, suppose that the measure $\nu_{\varphi, \psi^{\prime}, q}$ is a $\frac{q}{p}(\alpha-p+2)$ Carleson measure. Let $W_{\varphi, \psi}$ be a bounded operator from $\mathcal{D}_{\alpha}^{p}$ into $\mathcal{D}_{\alpha}^{q}$. Then $W_{\varphi, \psi}$ is compact from $\mathcal{D}_{\alpha}^{p}$ into $\mathcal{D}_{\alpha}^{q}$ if and only if

$$
\lim _{|a| \rightarrow 1} \sup \int_{\mathbf{D}}\left(\frac{1-|a|^{2}}{|1-\bar{a} z|^{2}}\right)^{\frac{q}{p}(\alpha+2)} d \mu_{\varphi, \psi \varphi^{\prime}, q}(\omega)=0
$$

and

$$
\lim _{|a| \rightarrow 1} \sup \int_{\mathbf{D}}\left(\frac{1-|a|^{2}}{|1-\bar{a} z|^{2}}\right)^{\frac{q}{p}(\alpha-p+2)} d \nu_{\varphi, \psi^{\prime}, q}(\omega)=0 .
$$

By using Theorem 2.14 and Theorem 2 of [5], we can prove the following theorem.

Theorem 3.6. Suppose that $0<p<q<\infty$ and $-1<\alpha<\infty$. Let $\varphi \in \mathcal{D}_{\alpha}^{p}$ be such that $\varphi$ is a self-map of $\mathbf{D}$ and let $\psi \in \mathcal{D}_{\alpha}^{q}$. For $p<\alpha+2$, suppose that the measure $\nu_{\varphi, \psi^{\prime}, q}$ is a $\frac{q}{p}(\alpha-p+2)$-Carleson measure. Suppose the operator $W_{\varphi, \psi}$ from $\mathcal{D}_{\alpha}^{p}$ into $\mathcal{D}_{\alpha}^{q}$ is bounded. Then there exists a constant $C>0$ such that

$$
\lim _{|a| \rightarrow 1} \sup I_{\varphi, \psi, \alpha} \leq\left\|W_{\varphi, \psi \varphi^{\prime}}\right\|_{e}^{q} \leq C \lim _{|a| \rightarrow 1} \sup I_{\varphi, \psi, \alpha}
$$

where

$$
I_{\varphi, \psi, \alpha}=\int_{\mathbf{D}}\left(\frac{1-|a|^{2}}{|1-\bar{a} \omega|^{2}}\right)^{(2+\alpha) q / p} d \mu_{\varphi, \psi \varphi^{\prime}, q}(\omega) .
$$

The following corollary is now immediate.

Corollary 3.7. Suppose that $0<p<q<\infty$ and $-1<\alpha<\infty$. Let $\varphi \in \mathcal{D}_{\alpha}^{p}$ be such that $\varphi$ is a self-map of $\mathbf{D}$ and let $\psi \in \mathcal{D}_{\alpha}^{q}$. For $p<\alpha+2$, suppose that the measure $\nu_{\varphi, \psi^{\prime}, q}$ is a vanishing $\frac{q}{p}(\alpha-p+2)$-Carleson measure. Then the operator $W_{\varphi, \psi}$ from $\mathcal{D}_{\alpha}^{p}$ into $\mathcal{D}_{\alpha}^{q}$ is compact if and only if

$$
\lim _{|a| \rightarrow 1} \sup \int_{\mathbf{D}}\left(\frac{1-|a|^{2}}{|1-\bar{a} \omega|^{2}}\right)^{(2+\alpha) q / p} d \mu_{\varphi, \psi \varphi^{\prime}, q}(\omega)=0 .
$$

Now we will deal with some results related to composition operators on Dirichlet type spaces.

Theorem 3.8. Suppose that $0<p<q<\infty$ and $-1<\alpha<\infty$. Let $\varphi \in \mathcal{D}_{\alpha}^{p}$ be such that $\varphi$ is a self-map of $\mathbf{D}$. Then the composition operator $C_{\varphi}$ from $\mathcal{D}_{\alpha}^{p}$ into $\mathcal{D}_{\alpha}^{q}$ is bounded if and only if the measure $\nu_{\varphi, q}$ is a $\frac{q}{p}(\alpha+2)$-Carleson measure for $L_{a}^{p, \alpha}$. 
Proof. Let $f_{n} \in \mathcal{D}_{\alpha}^{p}$ and let $g_{n} \in L_{a}^{p, \alpha}$ be such that $f_{n}^{\prime}=g_{n}$ and $g_{n}(0)=0$. First suppose that $C_{\varphi}$ from $\mathcal{D}_{\alpha}^{p}$ into $\mathcal{D}_{\alpha}^{q}$ is bounded. Also $C_{\varphi}$ from $\mathcal{D}_{\alpha}^{p}$ into $\mathcal{D}_{\alpha}^{q}$ is bounded if and only if there is a constant $C>0$ such that

$$
\left\|C_{\varphi} f_{n}\right\|_{\mathcal{D}_{\alpha}^{q}}^{q} \leq C\left\|f_{n}\right\|_{\mathcal{D}_{\alpha}^{p}}^{q}
$$

By using Lemma 2.2, we have

$$
\left\|C_{\varphi} f_{n}\right\|_{\mathcal{D}_{\alpha}^{q}}^{q}=\int_{\mathbf{D}}\left|f_{n}^{\prime}(w)\right|^{q} d \nu_{\varphi, q}(\omega) \leq C\left\|f_{n}^{\prime}\right\|_{L_{a}^{p, \alpha}}^{q}
$$

Thus

$$
\int_{\mathbf{D}}\left|g_{n}(w)\right|^{q} d \nu_{\varphi, q}(\omega) \leq C\left\|g_{n}\right\|_{L_{a}^{p, \alpha}}^{q} .
$$

Therefore by using Theorem 2.3, we get that the measure $\nu_{\varphi, q}$ is a $\frac{q}{p}(\alpha+2)$-Carleson measure for $L_{a}^{p, \alpha}$.

Similarly, we can prove the following result.

Theorem 3.9. Suppose that $0<p<q<\infty$ and $-1<\alpha<\infty$. Let $\varphi \in \mathcal{D}_{\alpha}^{p}$ be such that $\varphi$ is a self-map of $\mathbf{D}$. Then the composition operator $C_{\varphi}$ from $\mathcal{D}_{\alpha}^{p}$ into $\mathcal{D}_{\alpha}^{q}$ is compact if and only if the measure $\nu_{\varphi, q}$ is a vanishing $\frac{q}{p}(\alpha+2)$-Carleson measure for $L_{a}^{p, \alpha}$.

Similarly, we can prove these results for the following cases:

(i) $0<p \leq 2$ and $\alpha=p-1$.

(ii) $0<p \leq 1,-1<\alpha<p-1$ and $p<q<\infty$.

(iii) $\alpha+1<p<\alpha+2$.

(iv) $0<p<q<\infty$ and $\alpha=p-1$.

Acknowledgements. The author is indebted to the referee for many valuable comments and pointing out many mistakes in the paper.

\section{References}

[1] R. Aulaskari, D. A. Stegenga, and J. Xiao, Some subclasses of BMOA and their characterization in terms of Carleson measures, Rocky Mountain J. Math. 26 (1996), no. 2, 485-506.

[2] M. D. Contreras and A. G. Hernández-Díaz, Weighted composition operators on Hardy spaces, J. Math. Anal. Appl. 263 (2001), no. 1, 224-233.

[3] C. C. Cowen and B. D. MacCluer, Composition operators on spaces of analytic functions, Studies in Advanced Mathematics, CRC Press, Boca Raton, FL, 1995.

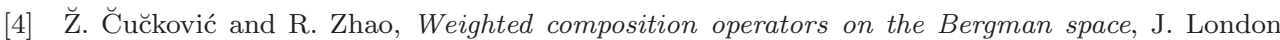
Math. Soc. (2) 70 (2004), no. 2, 499-511. 
[5] Weighted composition operators between different weighted Bergman spaces and different Hardy spaces, Illinois J. Math. 51 (2007), no. 2, 479-498 (electronic).

[6] T. M. Flett, The dual of an inequality of Hardy and Littlewood and some related inequalities, J. Math. Anal. Appl. 38 (1972), 746-765.

[7] D. Girela and J. Á. Peláez, Carleson measures, multipliers and integration operators for spaces of Dirichlet type, J. Funct. Anal. 241 (2006), no. 1, 334-358.

[8] P. R. Halmos, Measure Theory, Graduate Texts in Mathematics, vol. 18, Springer-Verlag, New York, 1974.

[9] R. A. Hibschweiler, Composition operators on Dirichlet-type spaces, Proc. Amer. Math. Soc. 128 (2000), no. 12, 3579-3586 (electronic).

[10] H. Koo and W. Smith, Composition operators between Bergman spaces of functions of several variables, Recent advances in operator-related function theory, Contemp. Math., vol. 393, Amer. Math. Soc., Providence, RI, 2006, pp. 123-131.

[11] R. Kumar and J. R. Partington, Weighted composition operators on Hardy and Bergman spaces, Recent advances in operator theory, operator algebras, and their applications, Oper. Theory Adv. Appl., vol. 153, Birkhäuser, Basel, 2005, pp. 157-167.

[12] D. H. Luecking, Forward and reverse Carleson inequalities for functions in Bergman spaces and their derivatives, Amer. J. Math. 107 (1985), no. 1, 85-111.

[13] _ Multipliers of Bergman spaces into Lebesgue spaces, Proc. Edinburgh Math. Soc. (2) 29 (1986), no. 1, 125-131.

[14] B. D. MacCluer and J. H. Shapiro, Angular derivatives and compact composition operators on the Hardy and Bergman spaces, Canad. J. Math. 38 (1986), no. 4, 878-906.

[15] M. J. Martín and D. Vukotić, Isometries of the Dirichlet space among the composition operators, Proc. Amer. Math. Soc. 134 (2006), no. 6, 1701-1705 (electronic).

[16] A. Montes-Rodríguez, The essential norm of a composition operator on Bloch spaces, Pacific J. Math. 188 (1999), no. 2, 339-351.

[17] Weighted composition operators on weighted Banach spaces of analytic functions, J. London Math. Soc. (2) 61 (2000), no. 3, 872-884.

[18] J. H. Shapiro, The essential norm of a composition operator, Ann. of Math. (2) 125 (1987), no. 2, 375-404.

[19] Composition operators and classical function theory, Universitext: Tracts in Mathematics, Springer-Verlag, New York, 1993.

[20] J. H. Shapiro and W. Smith, Hardy spaces that support no compact composition operators, J. Funct. Anal. 205 (2003), no. 1, 62-89.

[21] W. Smith, Brennan's conjecture for weighted composition operators, Recent advances in operator-related function theory, Contemp. Math., vol. 393, Amer. Math. Soc., Providence, RI, 2006, pp. 209-214.

[22] M. Tjani, Compact composition operators on some Möbius invariant Banach spaces, Ph.D. Thesis, Michigan State University, 1996.

[23] E. Wolf, Weighted composition operators between weighted Bergman spaces and weighted Banach spaces of holomorphic functions, Rev. Mat. Complut. 21 (2008), no. 2, 475-480.

[24] Z. Wu and L. Yang, Multipliers between Dirichlet spaces, Integral Equations Operator Theory 32 (1998), no. 4, 482-492.

[25] K. H. Zhu, Operator theory in function spaces, Monographs and Textbooks in Pure and Applied Mathematics, vol. 139, Marcel Dekker Inc., New York, 1990.

[26] N. Zorboska, Composition operators on weighted Dirichlet spaces, Proc. Amer. Math. Soc. 126 (1998), no. 7, 2013-2023. 